\title{
Cannabis Intoxication: What about Posterior Reversible Encephalopathy
} Syndrome?

\author{
Ouissal Aissaoui*, Kamal Seddiki, Karim Mediouni, Loubna Boujmai, Imane Mahdar, Samira Kalouch, Khalid Yaqini, and \\ Abdelaziz Chlilek
}

Department of Pediatric ICU, University Hospital of Casablanca, Morocco

${ }^{*}$ Corresponding author: Ouissal Aissaoui, Department of Pediatric ICU, University hospital of Casablanca, Morocco, E-mail: aissaoui.wissal@gmail.com

Received: 03 Sep, 2019 | Accepted: 19 Feb, 2020 | Published: 25 Feb, 2020

Citation: Aissaoui O, Seddiki K, Mediouni K, Boujmai L, Mahdar I, et al. (2020) Cannabis Intoxication: What about Posterior Reversible Encephalopathy Syndrome? Clin Res Open Access 6(1): dx.doi.org/10.16966/2469-6714.155

Copyright: (C) 2020 Aissaoui O, et al. This is an open-access article distributed under the terms of the Creative Commons Attribution License, which permits unrestricted use, distribution, and reproduction in any medium, provided the original author and source are credited.

\section{Abstract}

Posterior Reversible Encephalopathy Syndrome "PRES" refers to a clinic-radiological disorder of reversible subcortical vasogenic brain oedema in patients with classic acute neurological symptoms. Many causes are identified including drugs intoxications. Recent publications have portrayed the correlation of cannabis to ischemic and haemorrhagic cerebrovascular diseases but not to PRES.

We herein present a case report of a 13 years old boy admitted in our paediatric ICU for a PRES potentially caused by cannabis intoxication.

\section{Introduction}

Posterior Reversible Encephalopathy Syndrome (PRES) is a constellation of clinical and radiological findings that has been recognized increasingly in the past decade among children admitted to a pediatric Critical Care Unit [1].

Many etiologies were identified including drug intoxication. Cannabis intoxication has been associated to acute cerebrovascular events such as strokes and hemorrhages; however, PRES was never reported as a complication of this intoxication.

We report a case of 13 years old boy, admitted in our pediatric ICU for coma and status epilepticus secondary to cannabis intoxication.

\section{Case Report}

A 13 years old boy was admitted to our pediatric ICU for status epilepticus leading to coma and respiratory failure. The initial examination showed an unconscious patient with a GCS $6 / 15$; decerebration movements; and no pupillary abnormalities. The vital constants monitoring showed a Heartbeat at 112 beat per minute, a respiratory rate at 40 cycle per minute, a pulse oximetry at $89 \%$, a temperature at $39^{\circ} \mathrm{C}$ and a blood pressure at $140 / 78 \mathrm{mmHg}$.

He was intubated following a Crush induction, using Propofol $4 \mathrm{mg} / \mathrm{Kg}$ and Rocuronium $0.8 \mathrm{mg} / \mathrm{Kg}$.

Continuous sedation was initiated using Midazolam $(100 \mu \mathrm{g} / \mathrm{Kg} /$ hour) and Fentanyl $(2 \mu \mathrm{g} / \mathrm{Kg} /$ hour$)$.

\section{He received}

- Phenobarbital: loading dose of $10 \mathrm{mg} / \mathrm{Kg}$ followed by entertaining dose of $5 \mathrm{mg} / \mathrm{Kg} /$ day.
- Paracetamol $15 \mathrm{mg} / \mathrm{Kg} / 6$ hours.

- Empiric antibiotherapy: Ceftriaxone $(100 \mathrm{mg} / \mathrm{Kg} /$ day $)$ and Gentamycin 3mg/Kg/day.

A cerebral CT scan was performed and showed cerebral edema. The CSL study was normal. The blood culture was negative. The Chest X-ray showed radiological images of pneumonia and the tracheobronchial aspirate study identified a Pseudomonas aeruginosa infection. We changed antibiotherapy prescription to Imipenem (20mg/Kg/8hours), Amikacin $15 \mathrm{mg} / \mathrm{Kg} /$ day, Vancomycin $30 \mathrm{mg} / \mathrm{Kg} /$ day. The toxicological screening showed the presence of Cannabis in urine samples.

The patient was a febrile on the $3^{\text {rd }}$ day of antibiotics. Sedation was interrupted. The patient showed no signs of neurological recovery. Another CT scan was performed showing no abnormalities. An MRI was performed identifying a posterior reversible encephalopathy syndrome "PRES".

In fact, MRI showed typical neuroradiologic presentations of posterior reversible encephalopathy syndrome as seen in (Figures1-4).

On Day 10, a tracheotomy was performed. The convulsions ceased under phenobarbital therapy.

On Day 20, the respiratory weening was successful.

On Day 48, the patient showed no signs of neurological progress. In fact, a vegetative state persisted, and the decerebration movement appeared simultaneal to pain stimulus. A follow up CT scan was performed. The latter showed no significant abnormalities. The patient died later on due to septic shock. 


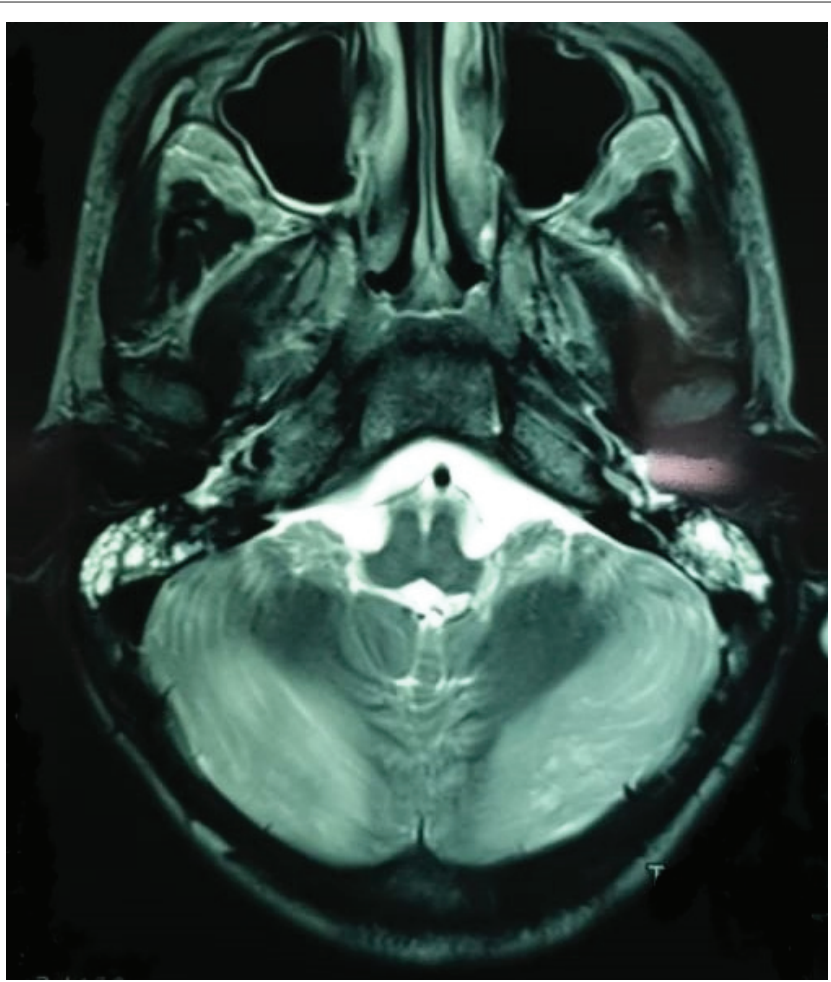

Figure 1: Areas of hyperintensities on axial T2 and fluid-attenuated inversion recovery involving bilateral cerebellar hemispheres.

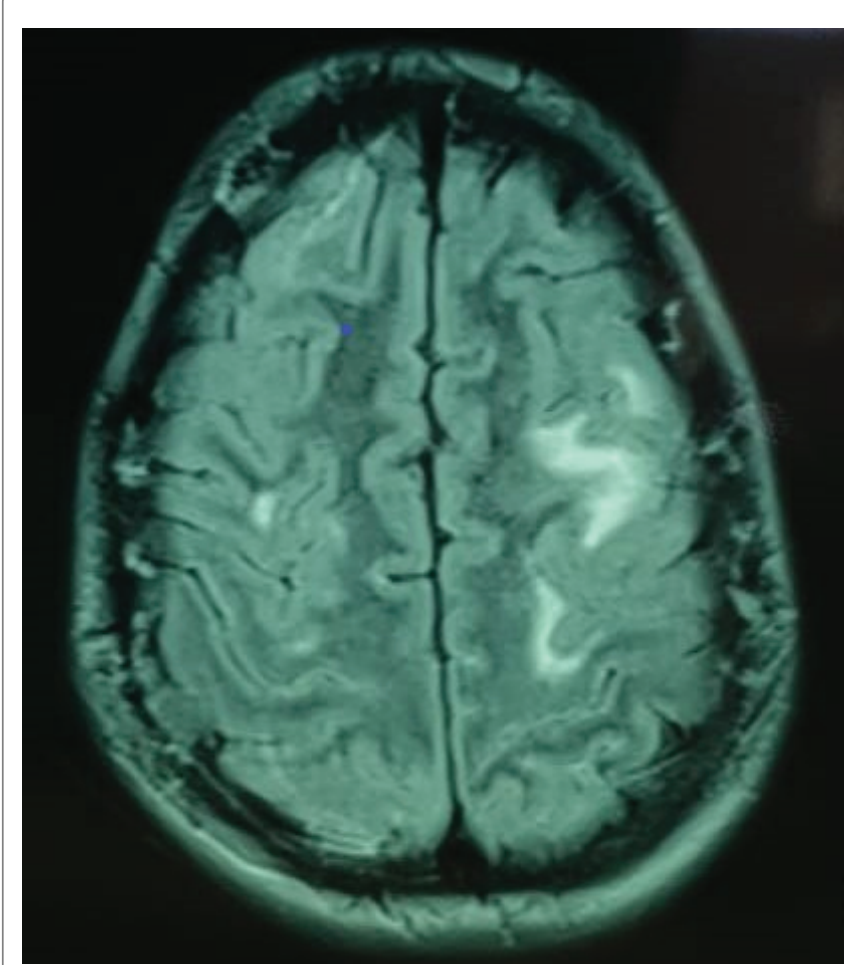

Figure 2: Lesions with the hyperintense signal on fluid-attenuated inversion recovery MRI locating in both subcortical white matter and cortical gray matter of bilateral parieto-occipital region.

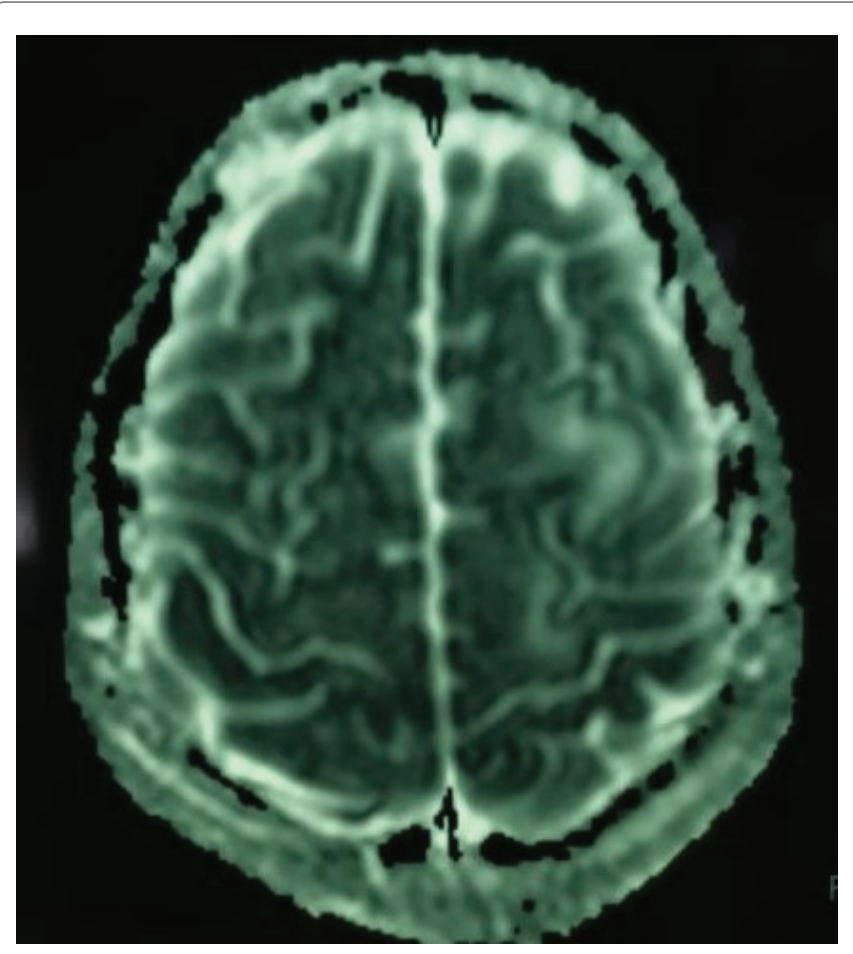

Figure 3: The involved regions appear as areas of $\mathrm{T} 1$ and $\mathrm{T} 2$ prolongation and are typically not restricted on DWI, reflecting vasogenic edema.

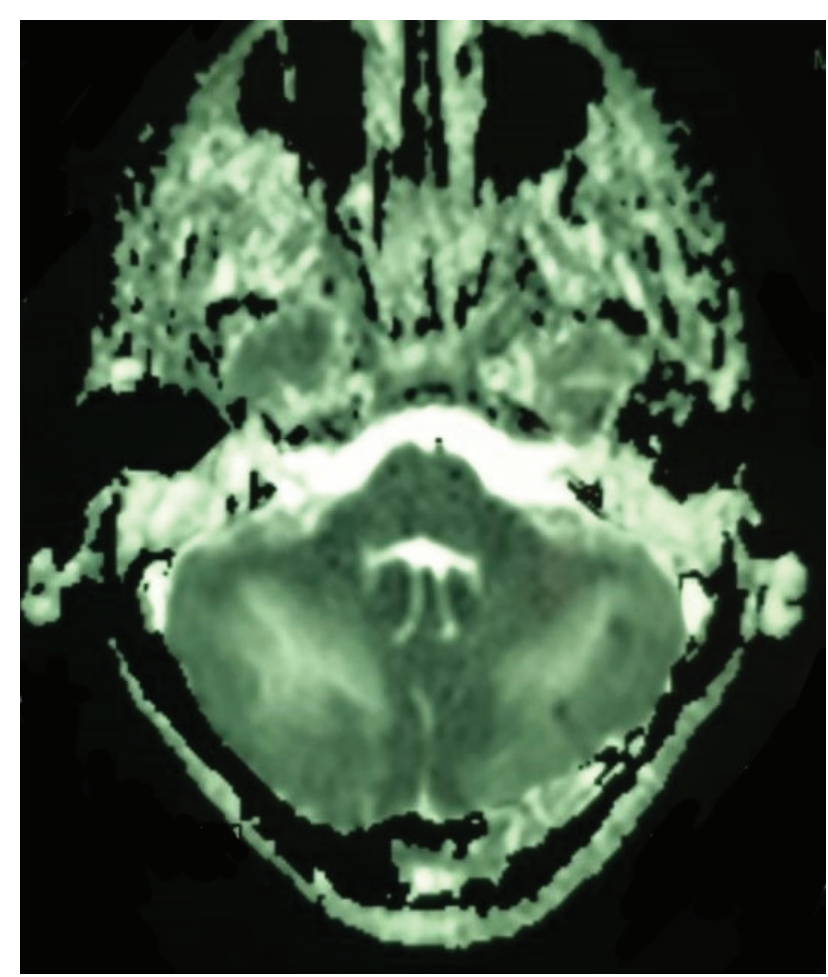

Figure 4: Axial FLAIR images and DWI showing bilateral areas of vasogenic edema affecting cortical and subcortical white matter in the parietal and occipital lobes, brain stem and cerebellum. 
We concluded to an "irreversible" PRES secondary to cannabis intoxication.

\section{Discussion}

PRES is a clinical syndrome with acute neurological findings resulting from disruption of the Blood-Brain Barrier and evidence of vasogenic edema on brain imaging [1].

Children are regarded to be more vulnerable to cerebrovascular dysfunction than adults because they have a narrower range of autoregulation in cerebral blood flow [2]. MRI findings in PRES typically include T2 and FLAIR hyperintensities in a parieto-occipital distribution [3].

The involved regions appear as areas of $\mathrm{T} 1$ and $\mathrm{T} 2$ prolongation and are typically not restricted on DWI, reflecting vasogenic edema [4].

Although PRES is known to be reversible, there in increasing data about cases with poor outcome.

For instance, in this retrospective study of PRES patients admitted to a consortium of 24 participating ICUs in France, the authors report a $16 \%$ fatality rate and a $37 \%$ rate of significant functional impairments [5]. The authors identified a sub-group as "Severe PRES" when the latter is associated with neurological failure which required intensive care management for monitoring or life support management. This subgroup is probably linked to a higher mortality and poorer outcome.

Regarding the involvement of cannabis intoxication, our literature review showed that acutely, cannabis alters global and regional cerebral blood flow and that could be the cause of vasogenic edema and PRES in our case.

Several studies on rat, mouse, rabbit, cat, and pig models were performed to examine the effect of cannabinoids on blood circulation as well as reactive vasodilation or vasoconstriction, particularly focusing on the cerebral vascular bed [6]. It was found that, both vasodilation and vasoconstriction have been observed following administration of cannabis in rodents [7]. Studies on acute neurovascular events related to cannabis use have appeared as early as 1964 (Mohan and Sood, 1964).

The 'vasogenic' theory is the more traditional hypothesis, which postulates that severe cerebral hyperperfusion would damage the brain blood barrier and cause cerebraledema and therefore PRES [8].

The other theories share a similar mechanism, where endothelial dysfunction causes resultant hypoperfusion and ischemia [9]. Cerebral vasoconstriction may then trigger this dysfunction.

Furthermore, many cases of ischemic strokes associated with cannabis smoking have been published since 1964. These strokes were more frequent in vertebrobasilar territory, suggesting susceptibility of posterior circulation [10].
On the other hand, a review about harmful cerebrovascular and neurological effects of smoking cannabis reported a total of 107 case reports involving cannabis intake and neurovascular complications that have been studied since 1964 to 2019 [7]. PRES did not figure among the cited complications, and therefore our case is the first report of PRES secondary to cannabis intoxication.

\section{Conclusion}

Cannabis intoxication is nowadays associated with multiple cerebrovascular effects, including ischemic strokes and hemorrhages. However, a PRES was never reported. We believe that this is the first case of PRES secondary to cannabis. The pathophysiology of the causality of cannabis in a PRES remains uncertain.

\section{References}

1. Raj S, Overby P, Erdfarb A, Ushay HM (2013) Posterior Reversible Encephalopathy Syndrome: Incidence and Associated factors in a Pediatric Critical Care Population. Pediatr Neurol 49: 335-339.

2. Kwon S, Koo J, Lee S (2001) Clinical spectrum of reversible posterior leukoencephalopathy syndrome. Pediatr Neurol 24: 361-364.

3. Fugate JE, Claassen DO, Cloft HJ, Kallmes DF, Kozak OS, et al. (2010) Posterior reversible encephalopathy syndrome: associated clinical and radiologic findings. Mayo Clin Proc 85: 427-432.

4. Feske SK (2011) Posterior reversible encephalopathy syndrome: a review. Semin Neurol 31: 202-215.

5. Legriel S, Schraub O, Azoulay E, Hantson P, Magalhaes E, et al. (2012) Determinants of Recovery from Severe Posterior Reversible Encephalopathy Syndrome. PLoS One 7: e44534.

6. Richter JS, Quenardelle V, Rouyer O, Raul JS, Beaujeux R, et al. (2018) A Systematic Review of the Complex Effects of Cannabinoids on Cerebral and Peripheral Circulation in Animal Models. Front Physiol 9: 622.

7. Archie SR, Cucullo L (2019) Harmful Effects of Smoking Cannabis: A Cerebrovascular and Neurological Perspective. Front Pharmacol 10: 1481.

8. Granata G, Greco A, lannella G, Granata M, Manno A, et al. (2015) Posterior reversible encephalopathy syndrome-Insight into pathogenesis, clinical variants and treatment approaches. Autoimmun Rev 14: 830-836.

9. Gao B, Lyu C, Lerner A, McKinney AM (2018) Controversy of posterior reversible encephalopathy syndrome: what have we learnt in the last 20 years? J Neurol Neurosurg Psychiatry 89: 14-20.

10. Bal S, Khurana D, Lal V, Prabhakar S (2009) Posterior circulation stroke in a cannabis abuser. Neurol India 57: 91-92. 